\title{
New 3D Objects Retrieval Approach using Multi Agent Systems and Artificial Neural Network
}

\author{
Basma Sirbal $^{1}$, Mohcine Bouksim $^{2}$, Khadija Arhid $^{3}$, Fatima Rafii Zakani $^{4}$, Taoufiq Gadi ${ }^{5}$ \\ Laboratory of Informatics, Imaging, and Modelling of Complex Systems (LIIMSC) \\ Faculty of Sciences and Techniques, Hassan 1st University \\ Settat, Morocco
}

\begin{abstract}
Content-based 3D object retrieval is a substantial research area that has drawn a significant number of scientists in last couple of decades. Due to the rapid advancement of technology, 3D models are more and more accessible yet it is hard to find, the models we are searching for. This created the need for efficient and robust retrieval methods, allowing the extraction of relevant matches from the human perspective. Hence, in this paper we are proposing a new framework for 3D object retrieval that starts with a pre-treatment consisting of an Artificial Neural Network (ANN) algorithm with Histogram of features, allowing us to extract a representative value for each category of the database. These values are used for the Multi Agents System (MAS). In this phase, we are classifying these categories according to their relevance to the request object. This sets a distinguishing weight for each object of the database allowing us to extract the right matches. Experiments have proven the stringent of this approach.
\end{abstract}

Keywords-3D object retrieval; $3 D$ image processing; distributed artificial intelligence; multi-agent systems; artificial neural network (ANN)

\section{INTRODUCTION}

The rapid development of computer techniques, 3D sensors and imaging devices has led to the rapid growth of rich information contained 3D models. Hence, they are more accessible in our daily lives. This induces the urgent need for efficient retrieval and recognition technologies. An excellent retrieval algorithm implies that the matches extracted belong to the same category, and are relevant from the human perception. This involves the representation of the 3D model by its geometrical, topological or other properties into a compact descriptor. The process of extracting the right matches requires two main steps:

Offline: also is the indexing phase. Where the proprieties of the models are exploited to represent it, a signature is computed for every object of the database, and stored for further usage. Any pre-treatment needed is executed in this phase.

Online: this second phase necessitate that the retrieval system takes a 3D object as input, then obtain the closest and more relevant matches for this query, its signature is computed using the chosen method, then distances between the signature of the request and those of the objects of the database are calculated and compared.

In this work, we propose a new content-based retrieval framework that exceeds the performance of well-known ones.
This approach is composed of three major phases; the first one is a pre-treatment employing an Artificial Neural Network (ANN), followed by a Multi agent system and finally the matches' extraction.

The agent notion has been introduced to ease the development of complex software and bring new solutions for unsolved issues. Still it has not been fully exploited [1]. The multi agent phase is where we are using the results generated by the ANN algorithm to classify the classes of the used database in order to extract values that are going to be used afterwards to refine the results of this retrieval process.

In this paper, we are answering following questions: How can we improve the quality and the relevance of the matches given by existing retrieval frameworks? Is it possible to exploit existing methods to achieve the aim?

This paper is structured as follows: Section 2 briefly reviews the related work and interesting work to mention, followed by the background in Section 3. In Section 4, we describe the proposed proposed approach. Experimental results and analysis are provided in Section 5. Finally, in the last section, conclusion and some perspectives are covered.

\section{RELATED WORK}

In the past decade, a number of content-based 3D model retrieval techniques have been developed. According to Johan, D. Tangelder et al. [2], these approaches can be sorted into 3 main categories, they also indicated that these categories are fusible since many of them can fit into more than one group. For more details about retrieval methods readers can refer to these surveys [3], [4] Each category of methods has advantages and disadvantages, hence why we decide to exploit methods from the two different categories we are discussing next.

Feature based methods: are based on geometric and topological features, extracted directly from the 3D model. Many scientists dedicated their work to this category. We address some interesting ones. To deal with queries of different modalities, Shah et al. [5] proposed using a different way of representing the 3D Model surface, Keypoints-based Surface Representation (KSR) technique involving the geometrical relationship between the detected 3D keypoints for local surface representation. Bouksim et al. [6] introduced a new approach, the heart of it is a multi-criteria method that generates a compact descriptor, using the Data envelopment analysis method (DEA) [7]. Tabia et al. [8] proposed a 3D shape descriptor based on local CNN features encoded using 
vectors of locally aggregated descriptors instead of conventional global CNN, using Convolutional Neural Networks (CNN) that includes all the entities from all modalities into a common space. Finally another interesting work is the one of Zeng and all [9], proposed a convolutional neural network based multi-feature fusion learning method for no rigid, using both the heat kernel signature (HKS) descriptor and the wave kernel signature (WKS) descriptor.

Geometry based methods (view based): The perception behind this category of methods is that two 3D models are matching, if they look similar from all viewing angles. This is accomplished by representing the 3D model using representative $2 \mathrm{D}$ captures. Many methods were published in the literature, a lot of them uses representations like binary images, projection or depth images. We are listing some of the methods existing in the literature. In their work, Wang et al. [10] introduced a boosting approach, where view's discriminative ability is analysed using the proposed reverse distance metric, then an algorithm introduced by the authors is employed to boost the multi-model graph learning based retrieval method. Another interesting work to mention, Lee and al [11] proposed a feature aggregation method, Cross-View Convolution (CVC), which models a 3D shape as a sequence of rendered views. Then used a Cross-Domain Triplet Neural Network (CDTNN) that incorporates an adaptation layer to match the features from different domains better and can be trained end-to-end. In another hand, Liu and al [12] propose a discriminative multi-view latent variable model (MVLVM) for 3D models retrieval. The MVLVM allows to have an undirected graph structure in which the view set of a given 3D object is treated as the observations from which to discover the latent visual and spatial contexts. Then, they use a learning and inference process of MVLVM for view-based 3D object retrieval.

\section{BACKGROUND}

In this section, we are discussing the methods and technologies that supports this research paper. Initially, we are starting with a technology that has proven its strength in many fields, it is Artificial Neural Network (ANN). We are exploiting it for the pre-treatment

\section{A. Artificial Neural Networks (ANNs)}

Artificial Neural Networks (ANNs) has been used for the purpose of $3 \mathrm{D}$ object retrieval for a couple of decades now. Here we are mentioning some interesting works, starting with Qayyum and al [13], where they are using deep convolutional neural network (CNN) that is trained for classification of medical images in order to upgrade existing content based medical image retrieval (CBMIR) systems. Furthermore Zhu and al [14] trained Convolutional Neural Network (CNN), and used the extracted features for 3D object representation. Bouksim and al [15] used a histogram of features extracted directly from the 3D along with an artificial neural network (ANN) algorithm for the training, the results of the hidden layers are then used as a descriptor in the retrieval system. For a detailed description of the technology we invite the readers to visit this e-book realised by Nielsen [16].
Next we are giving a brief introduction of the core technology used for this research, Multi Agents System (MAS).

\section{B. Multi Agent System (MAS)}

Multi-agent systems (MAS) have been the interest of more and more authors, since it provides adequate solutions for many complicated issues in several domains. It has been explored in a large number of software related domains as robotics, sustainable energy distribution [17], [18], but also in more general fields such as psychology [19] or biology [20]. The agent paradigm shifts different operative implementations. The more common ones are management agents and simulation agents; for in depth details you can consult the work of [21], [22].

Moreover, we are listing some commonly used methods in the literature that consolidates the proposed work. We adopted three methods that are based around strong mathematical Models.

\section{Featured Methods}

We experimented with different methods before agreeing on this composition that serves the purpose of refining the results existing in the literature. We are giving an overview of the approaches, we invite the readers to read the papers referred for more details.

DEA: Bouksim et al. [6], provided an approach for retrieval the core of it is a multi-criteria method that generates a compact descriptor, which represents the signature for each $3 \mathrm{D}$ model. The main intention behind this approach is to exploit the best out of each criterion (i.e., measure) by extracting a combined score using the Data envelopment analysis method (DEA), also known as frontier analysis introduced by Charnes, Cooper [7], and Rhodes in 1978. It is a linear programming method, which hypothetically measures the efficiency of the decision-making units (or DMUs) when this later present multiple inputs and/or outputs.

PANORAMA: Introduced by Papadakis and Al [23] is a $3 \mathrm{D}$ shape descriptor, initially utilizes a set of panoramic views of the $3 \mathrm{D}$ object, this allows to describe the position and orientation of the object's surface in 3 dimensional space. They acquire a panoramic view of the $3 \mathrm{D}$ object by projecting it to the lateral surface of a cylinder parallel to one of its three principal axes also situated at the centroid of the object. Later the object is projected to three perpendicular cylinders, each one of them is aligned with one of its principal axes in order to capture the global shape of the 3D object. For every projection they calculate the corresponding 2D Discrete Fourier Transform as well as 2D Discrete Wavelet Transform. They further increase the retrieval performance by employing a local (unsupervised) relevance feedback technique that shifts the descriptor of an object closer to its cluster centroid in feature space.

Light Field: Chen et al. [24] provided a visual similaritybased 3D Object retrieval system, it calculates the similarity between $3 \mathrm{D}$ objects by visual similarity. The primary idea is that if two 3D Objects are similar, they should look similar from all viewing angles. A hundred orthogonal projections of each object, disregarding symmetry, are coded both using 
Zernike moments and Fourier descriptors as features for the retrieval process.

In the following section we are giving a visual overview of this framework., followed by a detailed description of the proposed approach.

\section{PROPOSED APPROACH}

Our intention is to get the best matches for a 3D Object request. Most of the existing databases are categorised into different classes. Therefore the request object must fit into one of them. This inspired us to believe that weighting the elements of each class of the database with a favouring value can optimize the retrieval process. In this work we are elaborating a new framework for 3D objects retrieval, composed of two main phases an offline pre-treatment and an online classification using a Multi Agent System (MAS) which will carry out the weighting, at a final step we are extracting the matches with the Panorama method [23].

Before giving more details about this approach, the first figure Fig. 1, represents the architecture of this approach.

As described in Fig. 1, this framework is composed of two main phases. An offline phase where the pre-treatment takes place, allowing to extract representative values for the database using an artificial neural network (ANN) algorithm. Afterwards comes the online phase with two steps. The first step is a classification of the categories of the database with a multi agent system, therefore extracting the favouring weight for every object. The second and last step is using these values to extract the matches using Panorama method [23].

\section{A. The Offline Phase}

Instead of using the whole data base we propose in this approach to extract a representative Object for each class of the used database. The first phase of the pre-treatment, consists of extracting a histogram of features directly from the 3D objects. Followed by a training phase using an artificial neural network (ANN) algorithm; this last point helps to train the ANN fast and with consistent data. Once trained it allows us to extract a representative object for each class of the database [15], [16].

Since we are using three methods in the classification phase we are computing the signatures for the representative objects in the database using two of the methods, Light Field [24] and DEA [6]. Then we are computing the signatures for all the database objects using the Panorama [23] since we are going to be using it both in the classification and in the extraction phase.

\section{B. The Online Phase}

In this phase the request $3 \mathrm{D}$ object is given by the user. How can we extract the best matches for this later?
First we need to order the classes of the database according to their relevance. This will allow us to get more accurate results.

Each retrieval method has advantages and disadvantages, hence why we are using the combination three different methods for an efficient classification. Thanks to the pretreatment we now get to use only the most representative object for each class. The best way is to parallelize the process, hence why we are implementing a Multi Agent System (MAS). Distributed artificial intelligence in the form of MAS allows the classification part of the framework to be faster and reliable.

This Multi Agent System's architecture is composed of three layers, each one involves agents with different tasks. We are describing it in what follows.

The first layer is composed of 19 agents for each method. Each one of them computes the distance between the signatures of the representative element computed offline using the method and the signature of the request object. This information is then communicated to the elements of the second layer.

The second layer involves three agents, one for each method. In this agent, the distances representing each class are ordered, giving a classification of nineteen classes according to their relevance in comparison to the request objects 'signature. Each of the classifications is transferred to the final layer consisting of one agent.

Since each method has a different way of indexing the 3D objects, this last agent exploits the results sent to it and normalizes the signatures given by the three methods values. Then it takes the averages of these values for a unified classification, these values representing each class are considered as the weights. Each of the objects in the database takes a weight according to the order given to the class they belong to.

Then comes the final part of the process. The same agent uses the signatures computed by the method Panorama from the offline phase, and computes the distances between each one and the request object. Simultaneously the final representative score is computed using this distance and the weights representing each object as follows.

$s_{i}=d_{i} e^{w_{i}}$

Where $\mathrm{Si}$ is the score for an element $\mathrm{i}$, and di is the distance between the element $i$ and the request object, wi is the weight computed based on the classification. The smaller the score the more relevant the match is, hence why we order them. This gives us the matches relevant for the request Object. This method has proven its strength as showed in the next section. 


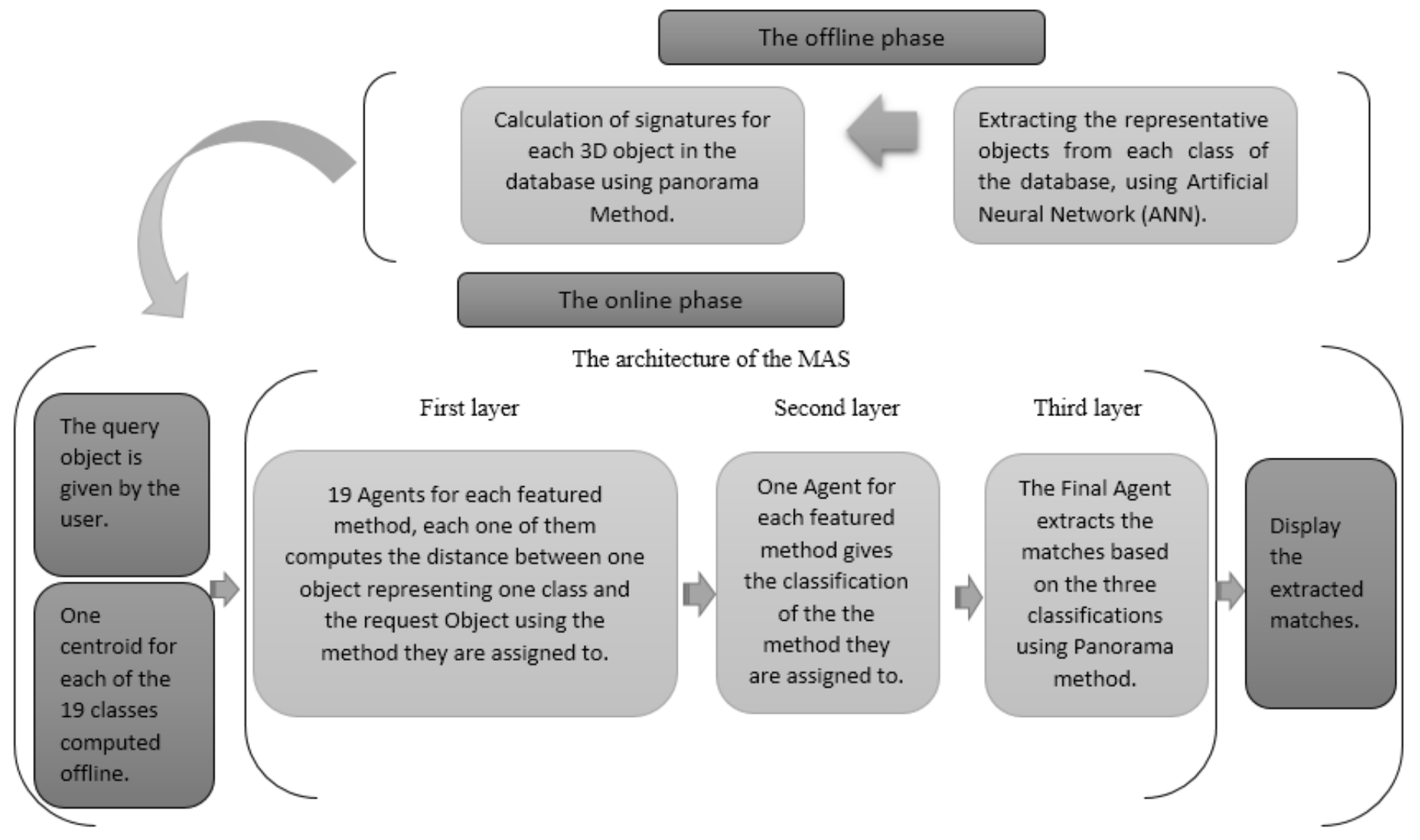

Fig. 1. The Architecture of the Proposed Framework.

\section{EXPERIMENTAL RESULTS}

In this section of this paper we are demonstrating the efficacy and the discriminative capacity of this approach through experimental results. We are comparing the results achieved with those of well-known methods: D2 [25], DEA [6], Harmonics [26], Panorama [23] and Light Field [24].

Foremost, we selected a reliable database to test the method. We choose to use Princeton's segmentation benchmark database [27].The selection of the database have been influenced by many criterions, some of them are the number and the diversity of the models. This database includes 380 3D models portioned into 19 classes (Human, Cup, Glasses, Airplane, Ant, Chair, Octopus, Table, Teddy, Hand, Plier, Fish, Bird, Armadillo, Bust, Mech, Bearing, Vase, and Fourleg).

The first experiment consists in computing the 10 nearest neighbours for each object using different methods, then we record the percentage of those that are right among them, whereas if the result belongs to the same class it is considered as relevant. Finally we obtain the accuracy for each Class by computing the average of all the results for each object of that class. Table 1 presents the results obtained from the proposed approach along with other known methods which are D2 distributions, 3D Harmonics, DEA, Panorama and LightField. We can observe from the results that our method exceeds all other methods, let's take for example the class Glasses, our approach obtained $98.5 \%$ of correct results, which is the highest of all results. Whereas for the class Ant our approach obtained $100 \%$ correct matches, same results were obtained for the class Teddy. We can also observe that our approach got the double of the results obtained by the other methods for the class Vase. Overall our method surpasses all the other methods except for the class ARMADILLO where two methods slightly surpasses ours, however our method extracted correctly all 7 neighbours for this class as illustrated in the third figure, Fig. 3.

We are carrying out with a commonly used test, precisionrecall diagrams. Recall is the ratio of relevant to the query retrieved models to the total number of relevant models while precision is the ratio of relevant to the query retrieved models to the number of retrieved models. The evaluations were performed by using each model of a dataset as a query on the remaining set of models and computing the average precisionrecall performance overall models. , all

$$
\begin{aligned}
& \text { Recall }=\frac{\text { relevant correctly retrieved }}{\text { all relevant }}, \\
& \text { Precision }=\frac{\text { relevant correctly retrieved }}{\text { all retrieved }} .
\end{aligned}
$$

The second figure, Fig. 2 illustrates the precision-recall graphs obtained for the proposed method along with DEA [6], Harmonics [26], Panorama [23] and Light Field [24].

We can clearly observe from the curves that the proposed approach surpasses all the other methods, this shows the capacity of this approach. 
TABLE I. The Performance of EACH OF THE Methods BASEd on the EXtraction of 10 NeARest Neighbours

\begin{tabular}{|c|c|c|c|c|c|c|}
\hline & Panorama & Light Field & DEA & Harmonics & D2 & The proposed approach \\
\hline Human & 79 & 56,5 & 52,5 & 41 & 47,5 & 100 \\
\hline CUP & 87,5 & 72,5 & 56,5 & 76,5 & 46 & 96 \\
\hline Glasses & 93,5 & 82 & 61,5 & 87 & 83 & 98.5 \\
\hline Airplane & 93 & 83,5 & 50,5 & 79 & 55 & 95 \\
\hline ANT & 97,5 & 79 & 98 & 54,5 & 42,5 & 100 \\
\hline CHAIR & 98,5 & 97 & 52,5 & 94 & 66 & 100 \\
\hline OCTOPUS & 57,5 & 70,5 & 54 & 41 & 20,5 & 95 \\
\hline TABLE & 93 & 57,5 & 75,5 & 45,5 & 42 & 98 \\
\hline TEDDY & 100 & 95,5 & 92,5 & 93 & 63 & 100 \\
\hline HAND & 78,5 & 37,5 & 52,5 & 32,5 & 29 & 94.5 \\
\hline PLIER & 92 & 94 & 93,5 & 63,5 & 69,5 & 100 \\
\hline FISH & 96 & 82 & 79,5 & 76,5 & 50 & 100 \\
\hline BIRD & 68,5 & 37,5 & 46,5 & 42,5 & 36,5 & 72 \\
\hline ARMADILLO & 95 & 63,5 & 93,5 & 54 & 26,5 & 89 \\
\hline BUST & 84,5 & 60 & 62 & 51 & 32,5 & 96 \\
\hline МЕCH & 77,5 & 85,5 & 79,5 & 82 & 53,5 & 96.5 \\
\hline BEARING & 81,5 & 54,5 & 33 & 40 & 22 & 89 \\
\hline VASE & 39,5 & 31,5 & 31,5 & 20,5 & 17,5 & 68.5 \\
\hline FOURLEG & 89,5 & 85,5 & 30,5 & 73,5 & 41,5 & 95.5 \\
\hline
\end{tabular}

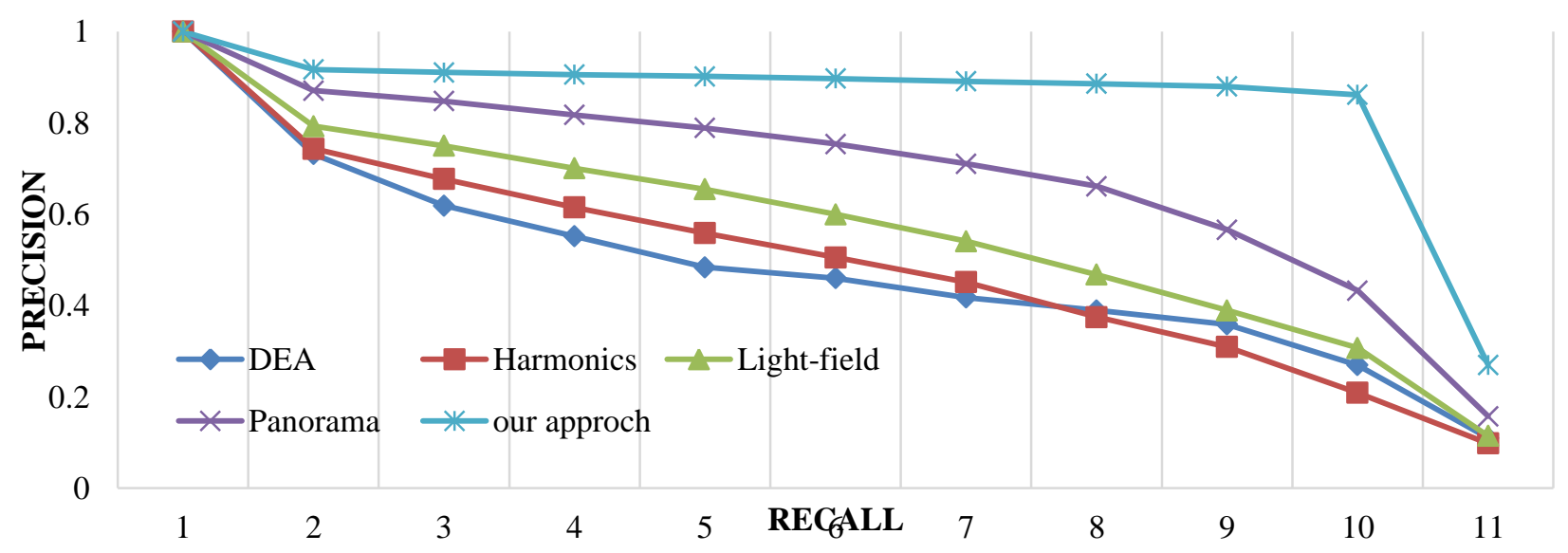

Fig. 2. Precision-Recall Graph using Four Different Descriptors with the Proposed One.

The third test will qualify the proposed method by computing some evaluation metrics, which are:

- Average Precision (AP): It is used to represent the precision performance of an Information Retrieval (IR) method over all relevant items. It is the average of precision values at each ranking position where a relevant item has been retrieved. For example, five relevant items are located at the following ranking positions: 1st, 2nd, 4th, 7th and 10th. Let the precision values at each one of these ranking position are: 1, 1, $0.75,0.57$ and 0.5 . Then, AP is the mean of these values (0.76).

- Average Dynamic Recall (ADR): The scalar is used to express the recall performance of an IR method at a given set of ranking positions. It is defined as:
$A D R=\frac{1}{R} \sum_{i=1}^{R} \frac{R 1(i)}{i}$

Where $\mathrm{R}$ indicates the lower ranking position to be included in the calculation (e.g. 20 first ranking positions), RI (i) represents the number of relevant retrieved items within the first i retrieved items.

- First Tier (FT) and Second Tier (ST): computes the recall for the top $\mathrm{C}-1$ and $2 *(\mathrm{C}-1)$ correctly retrieved objects in the result list, where $\mathrm{C}$ represents the number of item in each class.

- Discounted Cumulative Gain (DCG): a scalar that focuses on the items that are correctly retrieved and are in the front of the results list, since generally, a low 
ranking position has a low probability to be discovered by the user.

- F-Measure: The F-Measure simply generates a measure that combines the recall and precision values to express the overall performance of the retrieval system. It is computed as follow:

FMeasure $=2 \times \frac{\operatorname{Pr} \text { ecision } \times \operatorname{Re} \text { call }}{\operatorname{Pr} \text { ecision }+\operatorname{Re} \text { call }}$.

You can observe from table 2 that the proposed method obtained the highest values, followed by Panorama[23] then comes respectively Light field [24], Harmonics [26] and finally comes DEA[6]. This is just a confirmation of the results achieved in the previous experiments. Overall, this confirms that our method surpasses all others.

Finally, we select a test that will illustrate the results visually. That is the extraction of the 7 nearest neighbours, for 7 different objects and this using two methods previously used along with our suggested method. Our final figure, Fig. 3 represents the results as follows: the proposed approach (bottom centre), DEA (top left) and Panorama (top right); the illustrations in the left column shows the query models (we choose 7 models randomly among the data base), while the columns on the right displays the closest matches within the used database.

From the visual results we can easily see that the new approach succeed to provide refined matches. Let's take the class human for instance, the methods we are comparing ours to be both giving neighbours that belongs to other classes, for example the class Airplane, Octopus and Armadillo when all the neighbours given by the proposed approach are from the class human. Another example is the Model Octopus where our results belong all to the correct class, whereas the matches given by the other methods contain models from other classes, Fourleg and Glasses. Overall our approach proves once more its strength.

TABLE II. THE SCALAR METRICS FOR EACH METHOD

\begin{tabular}{|l|l|l|l|l|l|l|}
\hline $\begin{array}{l}\text { Descriptors / Scalar } \\
\text { Metrics }\end{array}$ & AP & ADR & FT & ST & DCG & F-Measure \\
\hline DEA & 0.45 & 0.14 & 0.40 & 0.28 & 5.98 & 0.27 \\
\hline Harmonics & 0.47 & 0.15 & 0.48 & 0.32 & 5.95 & 0.30 \\
\hline LightField & 0.54 & 0.16 & 0.55 & 0.35 & 6.21 & 0.32 \\
\hline PANORAMA & 0.66 & 0.18 & 0.69 & 0.41 & 6.62 & 0.35 \\
\hline Proposed approach & $\mathbf{0 . 8 1}$ & $\mathbf{0 . 2 0}$ & $\mathbf{0 . 8 8}$ & $\mathbf{0 . 4 9}$ & $\mathbf{7 . 0 3}$ & $\mathbf{0 . 3 6}$ \\
\hline
\end{tabular}

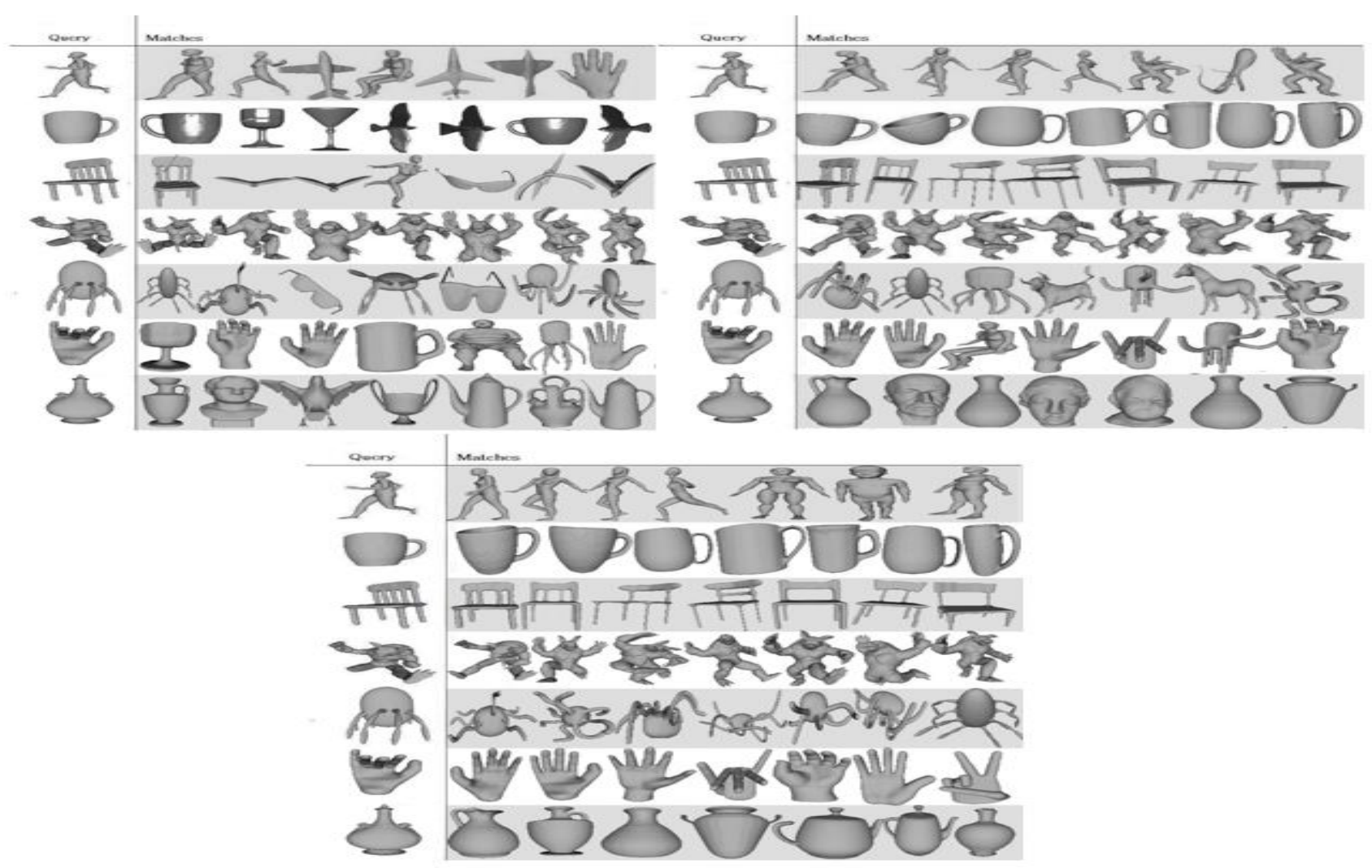

Fig. 3. Top 7 retrieved 3D models using DEA[6] (top left), Panorama[23] (top right) and the proposed approach (bottom centre). 


\section{CONCLUSION}

To sum up the most important aspects of our work, it introduces a new framework that utilizes Artificial intelligence to refine the matches retrieved for $3 \mathrm{D}$ objects. The process starts offline with a pre-treatment, employing an Artificial Neural Network algorithm, providing a representative value for each class of the used database followed by a Multi Agent System allowing us to classify therefore give a differentiating weight to each object of the database. Finally, we are using these values to extract matches for the request Object. Our method proves its potency in each one of the experiment it has been through. Overall, the proposed method surpasses some well-known methods and gives very satisfactory results. For our future work, we are experimenting with ways to use the same framework for 3D partial matching.

\section{REFERENCES}

[1] G. Jezic, Y.-H. J. Chen-Burger, R. J. Howlett, L. C. Jain, L. Vlacic, and R. Šperka, Agents and Multi-Agent Systems: Technologies and Applications 2018: Proceedings of the 12th International Conference on Agents and Multi-Agent Systems: Technologies and Applications (KESAMSTA-18). Springer, 2018.

[2] J. W. H. Tangelder and R. C. Veltkamp, 'A survey of content based 3D shape retrieval methods', Multimed. Tools Appl., vol. 39, no. 3, pp. 441471, Sep. 2008.

[3] K. Srinivasa Reddy, A. R, K. Kalaivani, and P. Swaminathan, 'A comprehensive survey on Content Based Image Retrieval system and its application in medical domain', Int. J. Eng. Technol., vol. 7, pp. 181185, Jan. 2018.

[4] G. Lara López, A. Peña Pérez Negrón, A. De Antonio Jiménez, J. Ramírez Rodríguez, and R. Imbert Paredes, 'Comparative analysis of shape descriptors for 3D objects', Multimed. Tools Appl., vol. 76, no. 5, pp. 6993-7040, Mar. 2017.

[5] S. A. A. Shah, M. Bennamoun, and F. Boussaid, 'Keypoints-based surface representation for 3D modeling and 3D object recognition', Pattern Recognit., vol. 64, pp. 29-38, Apr. 2017.

[6] Hassan 1st University et al., 'New Approach for 3D Mesh Retrieval Using Data Envelopment Analysis', Int. J. Intell. Eng. Syst., vol. 11, no. 1, pp. 1-10, Feb. 2018.

[7] A. Charnes, W. W. Cooper, and E. Rhodes, 'Measuring the efficiency of decision making units', European Journal of Operational Research, p. Vol 2 429-444, 1978.

[8] H. Tabia and H. Laga, 'Learning shape retrieval from different modalities', Neurocomputing, vol. 253, pp. 24-33, Aug. 2017.

[9] H. Zeng, Y. Liu, S. Li, J. Che, and X. Wang, 'Convolutional Neural Network Based Multi-feature Fusion for Non-rigid 3D Model Retrieval', p. 15,2018 .
[10] D. Wang, B. Wang, S. Zhao, H. Yao, and H. liu, 'View-based 3D object retrieval with discriminative views', Neurocomputing, vol. 252, pp. 5866, Aug. 2017.

[11] T. Lee, Y.-L. Lin, H. Chiang, M.-W. Chiu, W. Hsu, and P. Huang, 'Cross-Domain Image-Based 3D Shape Retrieval by View Sequence Learning', in 2018 International Conference on $3 D$ Vision (3DV), Verona, pp. 258-266, 2018.

[12] A.-A. Liu, W.-Z. Nie, and Y.-T. Su, '3D Object Retrieval Based on Multi-View Latent Variable Model', IEEE Trans. Circuits Syst. Video Technol., pp. 1-1, 2018.

[13] A. Qayyum, S. M. Anwar, M. Awais, and M. Majid, 'Medical image retrieval using deep convolutional neural network', Neurocomputing, vol. 266, pp. 8-20, Nov. 2017.

[14] Z. Zhu, C. Rao, S. Bai, and L. J. Latecki, 'Training convolutional neural network from multi-domain contour images for 3D shape retrieval', Pattern Recognit. Lett., Sep. 2017.

[15] M. Bouksim, K. Arhid, F. R. Zakani, M. Aboulfatah, and T. Gadi, 'New Approach for 3D Mesh Retrieval Using Artificial Neural Network and Histogram of Features', p. 11, 2018.

[16] M. Nielsen, 'Neural Networks and Deep Learning', p. 224, 2015.

[17] S. Howell, Y. Rezgui, J.-L. Hippolyte, B. Jayan, and H. Li, 'towards the next generation of smart grids: Semantic and holonic multi-agent management of distributed energy resources', Renew. Sustain. Energy Rev., vol. 77, pp. 193-214, Sep. 2017.

[18] A. K. Mbodji, M. L. Ndiaye, and P. A. Ndiaye, 'Decentralized control of the hybrid electrical system consumption: A multi-agent approach', Renew. Sustain. Energy Rev., vol. 59, pp. 972-978, Jun. 2016.

[19] G. Carslaw, 'Agent based modelling in social psychology', p. 267.

[20] R. Bardini, G. Politano, A. Benso, and S. Di Carlo, 'Multi-level and hybrid modelling approaches for systems biology', Comput. Struct. Biotechnol. J., vol. 15, pp. 396-402, 2017.

[21] D. Kremmydas, I. N. Athanasiadis, and S. Rozakis, 'A review of Agent Based Modeling for agricultural policy evaluation', Agric. Syst., vol. 164, pp. 95-106, Jul. 2018.

[22] S. V. Albrecht and P. Stone, 'Autonomous agents modelling other agents: A comprehensive survey and open problems', Artif. Intell. vol. 258, pp. 66-95, May 2018.

[23] P. Papadakis, I. Pratikakis, T. Theoharis, and S. Perantonis, 'PANORAMA: A 3D Shape Descriptor Based on Panoramic Views for Unsupervised 3D Object Retrieval', Int. J. Comput. Vis., vol. 89, no. 23, pp. 177-192, Sep. 2010.

[24] D.-Y. Chen, X.-P. Tian, Y.-T. Shen, and M. Ouhyoung, 'On Visual Similarity Based 3D Model Retrieval', Comput. Graph. Forum, vol. 22, no. 3, pp. 223-232, Sep. 2003.

[25] R. Osada, T. Funkhouser, B. Chazelle, and D. Dobkin, 'Shape distributions', ACM Trans. Graph., vol. 21, no. 4, pp. 807-832, Oct. 2002.

[26] T. Funkhouser et al., 'A search engine for 3D models', ACM Trans. Graph., vol. 22, no. 1, pp. 83-105, Jan. 2003.

[27] X. Chen, A. Golovinskiy, and T. Funkhouser, 'A Benchmark for 3D Mesh Segmentation', p. 12, 2009. 